\title{
A Simple Experiment to Explore Standing Waves in a Flexible Corrugated Sound Tube
}

Maria Eva Amorim, Teresa Delmira Sousa, and P. Simeão Carvalho, IFIMUP, Departamento de Física e Astronomia, Faculdade de Ciências, Universidade do Porto, Portugal

Adriano Sampaio e Sousa, Escola Secundária de Fontes Pereira de Melo, Porto, Portugal

S ound tubes, pipes, and singing rods are used as musical instruments and as toys to perform amusing experiments. In particular, corrugated tubes present unique characteristics with respect to the sounds they can produce; that is why they have been studied so intensively, both at theoretical and experimental levels. ${ }^{1-4}$ Experimental studies usually involve expensive and sophisticated equipment that is out of reach of school laboratory facilities. ${ }^{3-6}$ In this paper we show how to investigate quantitatively the sounds produced by a flexible sound tube corrugated on the inside by using educational equipment readily available in school laboratories, such as the oscilloscope, the microphone, the anemometer, and the air pump. We show that it is possible for students to study the discontinuous spectrum of sounds produced by a flexible corrugated tube and go even further, computing the speed of sound in air with a simple experimental procedure.

\section{Theory}

When air flows across an open-ended tube, a standing air pressure wave is formed with a frequency that depends on the length of the tube. For an open-end air column, the fundamental standing wave (1st harmonic) has pressure nodes (therefore, air displacement antinodes) at both ends and a

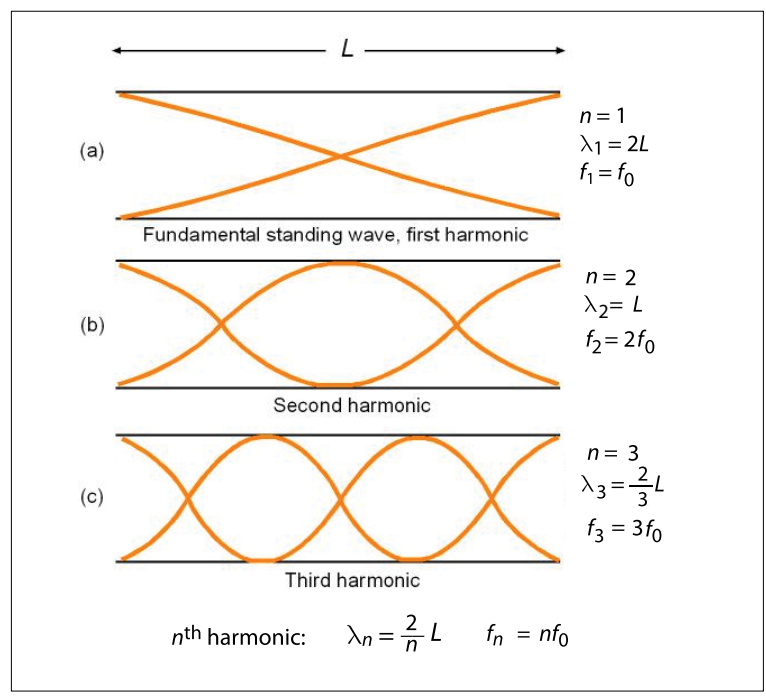

Fig. 1. Scheme of air displacement standing waves in a tube, corresponding to the (a) first harmonic, (b) second harmonic, and (c) third harmonic. General expressions for the wavelength and frequency of the $n^{\text {th }}$ harmonic are also shown. pressure antinode (displacement node) in the middle of the tube. $^{7-9}$ The length $L$ of the tube corresponds, in this case, to $L=\frac{\lambda_{0}}{2}$, where $\lambda_{0}$ is the wavelength of the standing wave. The corresponding frequency $f_{0}$ of the standing wave can be measured with an oscilloscope, capturing the sound with a microphone. High-order standing waves can also be formed, each one with a frequency that is a multiple of the fundamental wave, as shown in Fig. 1.

Relating $\lambda_{n}$ with $f_{n}$, where $n$ is the order of the harmonic standing wave, we can calculate the speed of sound in air,

$$
v_{\text {sound }}=2 L f_{0} \text {. }
$$

\section{Experimental procedure and results}

Sound tubes (and pipes) produce sounds when air goes through them. ${ }^{10}$ In flexible sound tubes corrugated on the inside, as the air flows over one ridge and then over another, it tumbles into a vortex. When the frequency of the vortex matches one of the natural resonant frequencies of the tube, sound is amplified. ${ }^{11}$ The faster the air flows through the tube, the higher the pitch of the sound produced by the vortex. ${ }^{4,12}$

When a flexible sound tube is spun in circular motion, there is a decrease of the air pressure $P$ at the free extremity,

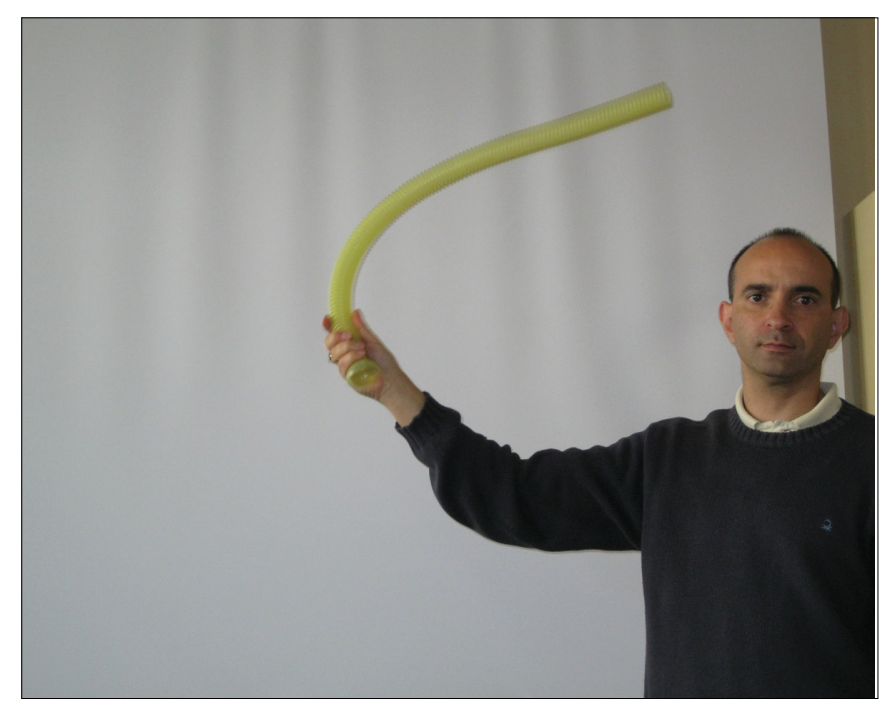

Fig. 2. Spinning of a flexible sound tube during a classroom demonstration. 
due to the speed it reaches, according to Bernoulli's law,

$$
P+\rho g h+\frac{1}{2} \rho v^{2}=\text { constant, }
$$

where $P$ is the fluid pressure (or energy per unit volume), $\rho g h$ is the potential energy per unit volume, and $\frac{1}{2} \rho v^{2}$ is the kinetic energy per unit volume. Therefore, a differential in air pressure is created between the extremity of the tube held by the hand (higher air pressure) and the free moving extremity (lower air pressure) (see Fig. 2), pushing air through the tube: the faster the tube spins, the higher the speed of air across it.

Such a situation was simulated in the laboratory by pumping air through one extremity of the tube so we could control the flux of air crossing it; at the other extremity, a digital anemometer Kestrel 1000 measured the speed of air, and a standard microphone captured the sound wave produced inside the tube. The electrical signal was then visualized in a digital oscilloscope Nicolet 310 and the period of the sound wave was measured, allowing us to know the corresponding frequency of the signal (see Fig. 3). Details of the experimental setup are shown in Fig. 4.

Results in Fig. 3 could also be obtained by replacing the oscilloscope in our setup in Fig. 4 with data acquisition software such as MacScope, PASCO Data Studio, Vernier Logger Pro, or Pico Technology's PicoScope, among others.

Figure 5 shows the frequency of the sound waves produced as a function of the air speed $v_{\text {air }}$ across the tube. It is easy to see that the frequency does not change continuously with $v_{\text {air }}$ but instead a well-defined sequence of steps is detected, corresponding to the different sounds that were heard.

By plotting the average frequency of each step as a function of the number of frequency steps detected $(N)$, we were able to determine the fundamental frequency $f_{0}$ corresponding to the first harmonic. Results are resumed in Fig. 6.

A careful analysis of the linear fit to the experimental data in Fig. 6 shows that the first step in Fig. 5 corresponds, in fact, to the second harmonic of the standing waves in the sound tube: the first harmonic was actually calculated from the slope of this straight line and is $f_{0}=(214 \pm 3) \mathrm{Hz}$. This sound could not be detected during the experiment; a similar situation was also reported in Ref. 4.

For an ideal open-ended tube, the speed of sound in air can be calculated using Eq. (1). However, as the air is moving in and out of the tube, the pressure variations do not drop to zero right at the open ends of the tube, but rather a small distance beyond. Therefore, the effective acoustic length $L^{\prime}$ is slightly greater than the physical length $L$ of the tube. For a cylindrical tube of radius $r$, a correction for the additional length must be introduced:4,14

$$
L^{\prime}=L+1.22 r .
$$

As the physical length of the sound tube is $L=75.0 \mathrm{~cm}$ and its radius $r=1.5 \mathrm{~cm}$, the acoustic length according to Eq. (3) is

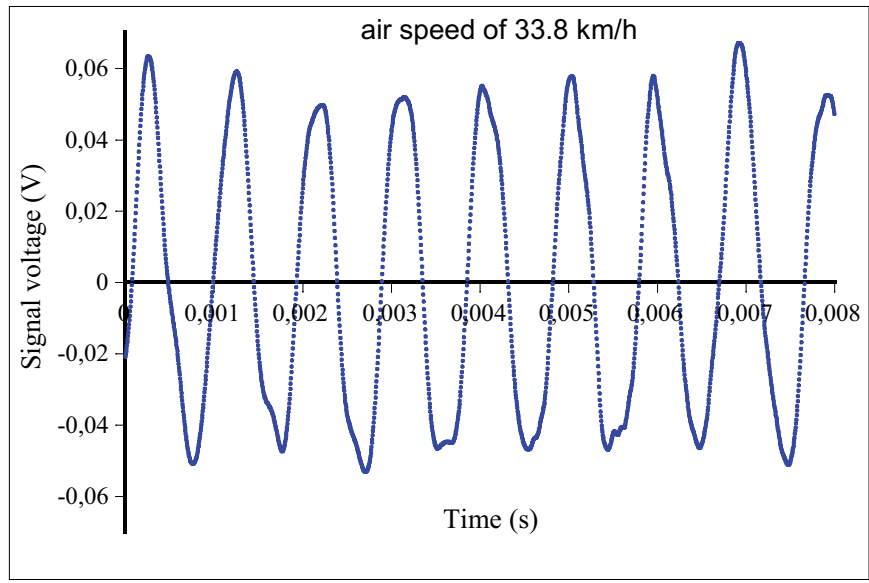

Fig. 3. Plot of the electric signal corresponding to the sound wave obtained for an air speed of $33.8 \mathrm{~km} / \mathrm{h}$.

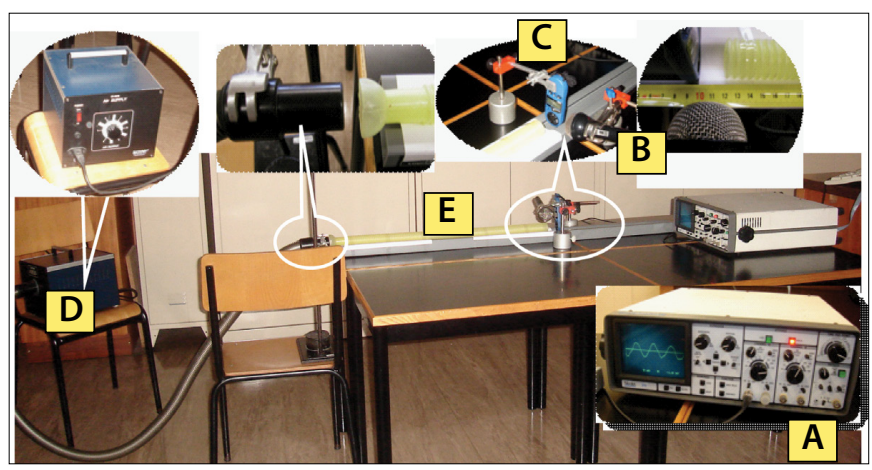

Fig. 4. Experimental setup: A - digital oscilloscope. The screen shows the electrical signal of a standing sound wave; B - detail of microphone and the anemometer, $2 \mathrm{~cm}$ away from the extremity of the sound tube. The ridges in the flexible tube can clearly be seen; $\mathbf{C}$ - wider view of the relative positions of the anemometer, the microphone, and the sound tube; D - air pump; E - the sound tube and a detail showing the position of the extremities of the air pump and the sound tube.

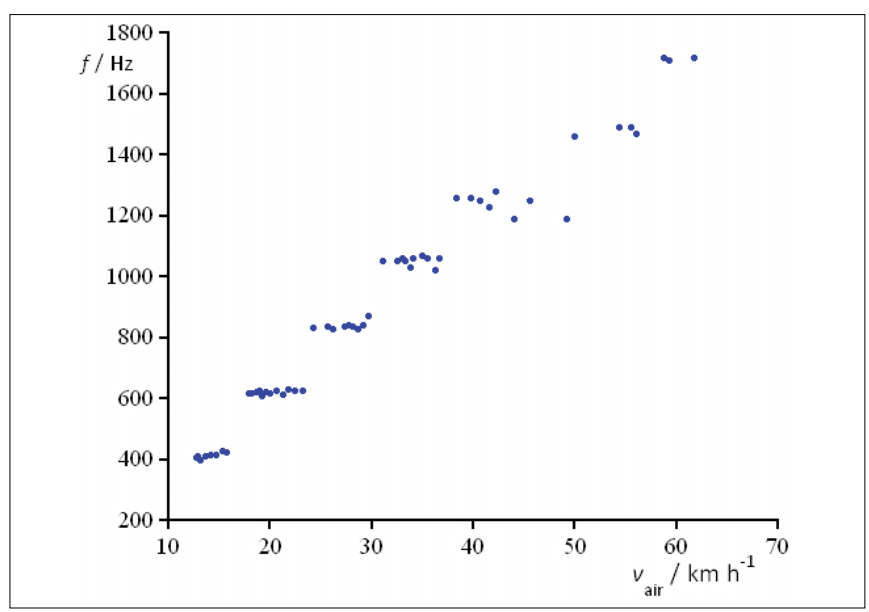

Fig. 5. Plot of the frequency of the standing sound waves as a function of the speed of air flowing through the tube. A discrete sequence of frequency steps can be clearly observed. 


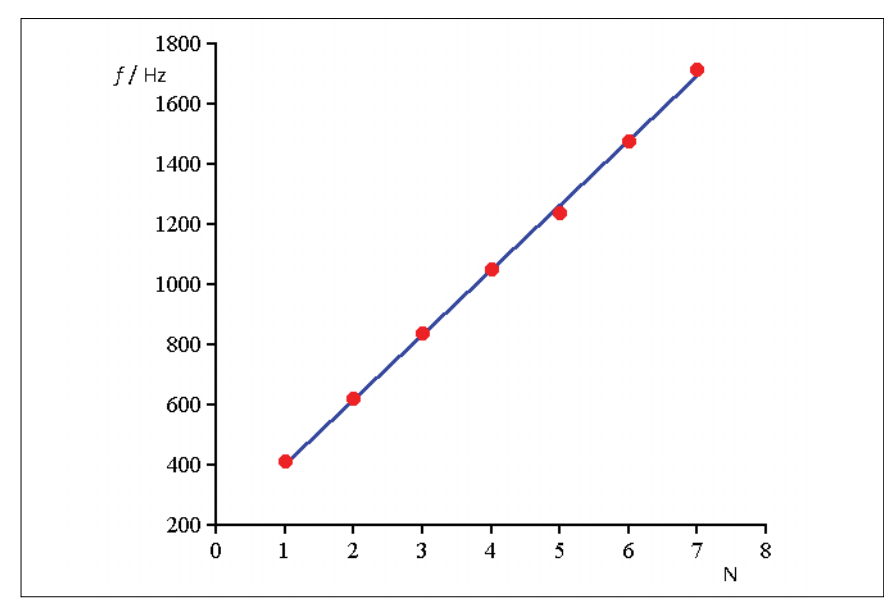

Fig. 6. Plot of the average frequency of the standing waves as a function of the number $N$ of frequency steps detected. The line represents the curve fit to the experimental data.

$L^{\prime}=76.8 \mathrm{~cm}$.

We are now able to calculate the speed of sound in air at experimental conditions, replacing $L$ with $L^{\prime}$ in Eq. (1):

$$
v_{\text {sound }}=(329 \pm 6) \mathrm{m} / \mathrm{s} \text {. }
$$

Just for a rough comparison, we can take as reference the speed of sound in air at $10^{\circ} \mathrm{C}$ and $50 \%$ humidity at the standard air pressure of $1 \mathrm{~atm},{ }^{13}$ which is $337.79 \mathrm{~m} / \mathrm{s}$. This means that the error is about $3 \%$, which is nearly within our experimental error.

It is worth mentioning that we have repeated this experiment using open-ended rubber tubes, one with a completely smooth inside and another with a spiral-shaped interior surface (a vacuum cleaner tube). In the former we only got one tone, while in the latter we noticed two harmonics. Therefore, it seems that the ridges along the flexible sound tube are truly responsible for the formation of the several harmonics we detected when air was pumped through the corrugated tube at different speeds.

\section{Conclusions}

With this simple experiment, students can investigate the different harmonics produced by a flexible tube corrugated on the inside. Although the use of an anemometer, if available, enables a quantitative measure of the air speed across the tube, a qualitative approach is enough for students to understand that the faster the tube is spun, the higher the air speeds across it. This is explained by Bernoulli's law and is a good context for teachers to explore that subject with their students.

The presence of a discontinuous spectrum of frequencies, characterized by well-defined frequency steps with an increasing sound pitch as the tube spins, is a clear indication of the formation of standing waves inside the sound tube. The analysis of experimental data supports motivation for a discussion about harmonics in sound waves and allows the calculation of the speed of sound in air with a reasonable accuracy.

\section{References}

1. V. Debut, J. Antunes, and M. Moreira, "A phenomenological model for sound generation in corrugated pipes," Presented at ISMA2007 - International Symposium on Musical Acoustics, September 9-12, Barcelona, Spain (2007).

2. V. Debut, J. Antunes, and M. Moreira, "Flow-acoustic interactions in corrugated pipes: time-domain simulation of experimental phenomena," in 9th International Conference on Flow Induced Vibrations (FIV-2008), edited by Zolotarev and Horacek, June 30- July 3, Prague, Czech Republic (2008).

3. Ulf R. Kristiansen and Geir A. Wiik, "Experiments on sound generation in corrugated pipes with flow," J. Acoust. Soc. Am. 121 (3), 1337 (2007).

4. Stefania Serafin and Juraj Kojs, “The voice of the dragon: A physical model of a rotation corrugated tube," Proceedings of the 6th International Conference on Digital Audio Effects (DAFx-03), September 8-11, London, England (2003).

5. V. Debut, J. Antunes, and M. Moreira, "Experimental study on the flow-excited acoustical lock-in in a corrugated pipe," Presented at ICSV14 - International Congress on Sound and Vibration, July 9-12, Cairns, Australia (2007).

6. Suriyan Laohalertdecha and Somchai Wongwises, "The effects of corrugation pitch on the condensation heat transfer coefficient and pressure drop of R-134a inside horizontal corrugated tube," Int. J. Heat Transfer 53, 2924-2931 (2010).

7. David R. Lapp, "Harmonics in an aluminum rod: 'A Golden Oldie,” Phys. Teach. 35, 314 (May 1997).

8. "Singing Rods," Arbor Scientific (2001); www.arborsci.com/ Data_Sheets/Files/SingingRods.pdf.

9. Hugh D. Young and Roger A. Freedman, University Physics, 9th ed. (Addison-Wesley, New York, 1996), pp. 633-634.

10. Suresh Chandra, "Resonance in an open tube: A new demonstration," Phys. Educ. 40 (6), 508 (2005).

11. Y. Nakamura et al., "Sound generation in corrugated tubes," Fluid Dyn. Res. 7, 255 (1991).

12. www.teachersource.com/SoundAndWaves/SoundWaves AndResonance/SoundTubes.aspx.

13. www.sengpielaudio.com/calculator-airpressure.htm.

14. Thomas D. Rossing, F. Richard Moore, and Paul A. Wheeler, The Science of Sound, 3rd ed. (Addison-Wesley, San Francisco, 2002), pp. 65-66. 